\title{
THE SANSEVERO CHAPEL, A THREE-DIMENSIONAL POINT OF VIEW
}

\author{
V. Cera ${ }^{1}$, D. Marcos González ${ }^{2}$, L. A. Garcia ${ }^{2 *}$ \\ ${ }^{1}$ Urban/Eco Interdepartmental Research Center, University of Naples Federico II, Italy - valeria.cera@unina.it \\ ${ }^{2}$ LFA Architectural Photogrammetric Laboratory, University of Valladolid, Spain - davmarcos.vll@gmail.com; \\ Lantonio@arq.uva.es
}

Commission II, WG II/8

KEY WORDS: architectural perspective, illusory space, three-dimensional survey, sensor integration, photogrammetry, laser scanner

\begin{abstract}
:
In this article, the importance of the three-dimensional survey in architectural spaces will be studied, taking special relevance in the study of the perception of perspective, since three-dimensional space would not be understood from a two-dimensional representation of space. The project aims to develop a comparison between the representation systems based on the automatic acquisition of various data by different 3D survey techniques. In particular, the document reports the results of an analysis based on the Sansevero Chapel in Naples.
\end{abstract}

\section{INTRODUCTION}

The traditional representation of architectural spaces is based on the orthogonal projection of the elements according to Cartesian axes, either dihedral projections or axonometric perspective systems. Although this type of representations gives us exhaustive knowledge of both the dimension and the scale, they do not provide in any case, the real perception of the object according from the point of view of the observer. This is the reason why the conical perspective is the best tool to achieve this goal.

When we talk about three-dimensional architectural surveys based on new technologies, such as the terrestrial laser scanner (TLS) or digital photogrammetry, we add to the representations of the traditional model, the spatial vision of spaces within the three-dimensional graphics environment, which allows to visualize in real time the three-dimensional model according from any point of view, and therefore the comparison of them. By this way, we can check the differences in perception of space between two-dimensional and three-dimensional representation. In the specific case of the Sansevero Chapel, this fact is particularly important, since both, its architecture and decoration are oriented to the vision from a specific point of the space, losing all its meaning when the observer changes his/her position.

In this article, we will make an extensive comparison between the different forms of representation of this space, as well as the different forms of perception of space according to the point of view of the observer. These points of comparison are extracted from the three-dimensional survey carried out and not from the direct vision of the observer, making this study extend to inaccessible architectures or already disappeared.

\section{RELATED WORKS}

The theme of architectural perspectives has fascinated researchers who have dedicated several studies over the years.

These are representations of architecture that, taking advantage of the linear perspective, the aerial perspective and other techniques, induce in the observer a perception of depth that breaks through the wall structure, expanding the space that hosts them to the limits of the eye. Their presence is ancient: perspectives of architectural subjects are already present in
Herculaneum, Pompeii, Paestum, Naples, Rome, in the houses of Augustus and Livia as in the Vesuvian ruins. They are still present throughout the Middle Ages, up until the Renaissance, when artist-scientists such as Filippo Brunelleschi, Leon Battista Alberti and Piero della Francesca experimented and theorized the laws of the costruzione legittima. They are the manifesto of the great optical and geometric knowledge of the ancients because they are a bridge that connects art with science.

In fact, illusionistic effects of such great power cannot be reached without an awareness of the laws of central projection and without a knowledge, at least empirical, of the complex mechanisms of visual perception. Therefore, a significant amount of research has investigated the architectural perspectives trying to explain the main aspects: how they were designed, from which observation point (one or multiples) they must be looked at in order to obtain the desired breakthrough effect, the correctness or otherwise of the geometric construction, the reason why a wrong perspective construction evokes a strong feeling of depth (Giordano et al., 2016; Rossi et al., 2018). To do this, the multiplicity of studies shows the fundamental role that the most advanced survey techniques play in the knowledge and documentation of the perspectives. Techniques ranging from laser scanning to high resolution photography that intertwine with a geometric analysis of the representations in an inverse process of the phases of perspective construction (Di Paola, 2014; Cabeleira, 2019).

\section{CASE STUDY}

The case study of this project is the Sansevero Chapel. Located in the historic center of Naples (Italy), the chapel is one of the most interesting museums in the city: the church - now deconsecrated - houses masterpieces such as the veiled Christ by Giuseppe Sanmartino, the Pudicizia by Antonio Corradini and the Disinganno by Francesco Queirolo. Other works of exquisite workmanship - such as anatomical machines - are exhibited here, helping to enhance the charm and uniqueness of the place.

In fact, the history of the chapel is linked with legends, family affairs and Masonry. According to a first legend, the church was erected on a pre-existing ancient temple dedicated to the goddess Isis. Another one, reported in 1623 by Cesare d'Engenio Caracciolo in Napoli Sacra, tells that its origins are linked to the 
Marian devotion of a man unjustly imprisoned. According to more recent studies, the chapel was conceived as a vow to the Virgin Mary of the Carafa della Spina - di Sangro family, following a tragic event. Whatever its origin, it is certain that the construction works of the church began in 1593 and from that moment on the chapel underwent progressively transformations by the noble Sansevero family, becoming its mausoleum in the 18th century. It was Raimondo di Sangro, seventh prince of Sansevero, who determined the major reorganization of the noble temple. The eighteenth-century restoration kept the perimeter dimensions, the simple architecture of the whole and the polychrome decoration of the apse unchanged. However, the transformation was consistent: the chapel - enlarged - has become a place where esoteric symbols merge with religious ones. The alchemical-scientific studies and the Masonic militancy of Raimondo di Sangro have transformed the church into a temple of the Masonry where every single work plays an irreplaceable role in the overall iconographic project imagined by the prince and probably unknown to the artists themselves. Therefore in the chapel everything acquires meaning only if the symbols and mysteries hidden in the marbles and paintings that adorn the temple are understood.
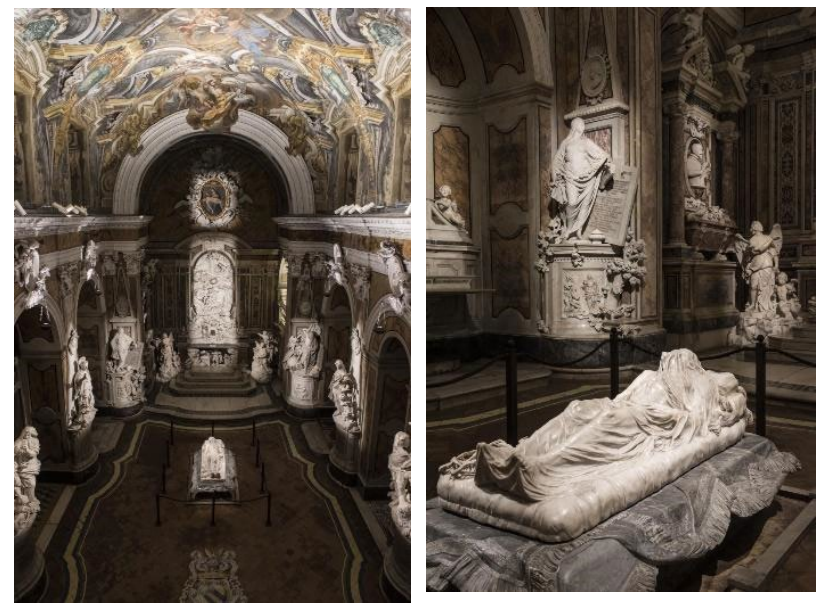

Figure 1. Sansevero Chapel

From the architectural point of view, the small church, typical of the Neapolitan baroque, has a rectangular shape and consists of a single nave. Along the side walls, eight round arches, four on each side, reveal the presence of the side chapels. A large arch separates the nave from the presbytery area, which is located at the end of the church and occupied by the high altar. The access to the sacristy and to the underground cavea is placed at the center of the two long sides. Above the arches the entire length of the chapel is crossed by a cornice. Above it, we can find a completely frescoed barrel vault. Signed and dated by Francesco Maria Russo, the fresco is known as Gloria del Paradiso or Paradiso dei di Sangro. It is one of the first works that the Prince of Sansevero commissioned for the chapel and reveals an interesting intertwining of symbolism and pictorial figuration. From a symbolic point of view, the triangle that - together with the dove - dominates the center of the scene has a particular interest. This geometric figure is in fact full of meanings: in the Christian universe it represents the Trinity, in the Pythagorean system the delta capital letter, with a triangular shape, is the symbol of cosmic birth, while in Masonic culture the sign is distinctive of the Venerable Master. From the pictorial point of view, the figurative structure is constructed with a strong perspective: fake three-dimensional architectures are reproduced on the twodimensional plane of the intrados of the vault. By virtue of the vanishing point, the perception of the scene is possible from a certain position of the observer. In the end, at the base of the vault, just above the cornice, there are six splayed windows that provide light to the chapel.

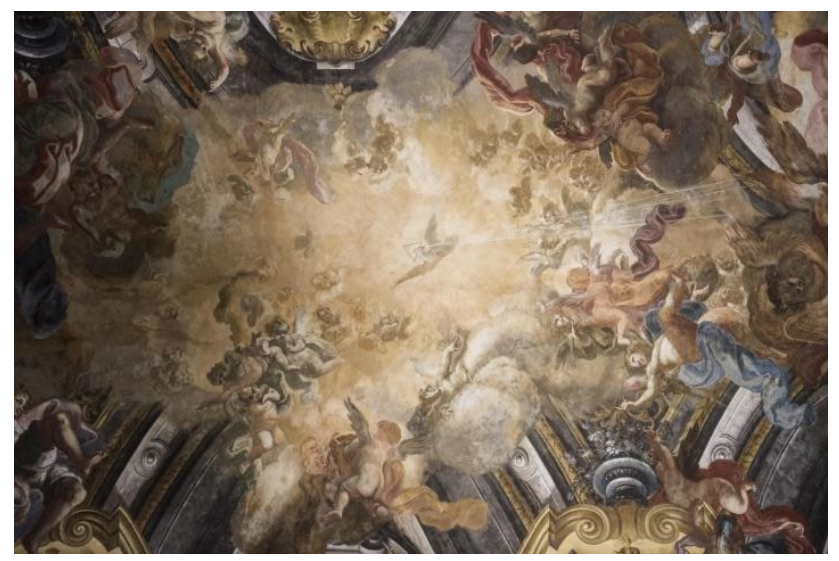

Figure 2. Sansevero Chapel. A detail of the vault's fresco

The rich symbolism present in the works and architecture of the Sansevero Chapel does not make possible a clear and univocal interpretation for its complexity. This is evident in the pictorial representation of the vault. The illusionistic layout of the imaginative architectures, the sudden gashes, the angels and the figures ideally converging towards the center make this case particularly significant for the study of the perception of threedimensional perspective representations transposed on planes.

\section{ARCHITECTURAL SURVEY}

In literature, multiple researches have defined the integration of several geomatics methods as the recognised acquisition protocol for the documentation of Cultural Heritage. 3D active sensors such as terrestrial laser scanning (TLS), 3D passive sensors such as terrestrial and aerial photogrammetry and mapping mobile systems have been described in their application in historic and cultural heritage environments. As known, each of these techniques has its own advantages and disadvantages in terms of precision, accuracy, costs, visual realism, acquisition and processing times (Cera et al, 2017; Calantropio et al., 2018; Ogawa et al., 2019). So the ideal approach is an integrated methodology in order to maximize single instrument performance. In this regard, recent years have seen significant improvements in the efficiency of automatic metric and colorimetric feature extraction systems (Farella et al., 2019; Pérez-Sinticala et al., 2019) as well as in the procedures for combining data from heterogeneous sources (Bracci et al., 2018; Liang et al., 2018; Toschi et al., 2018). In this way it is possible to obtain, starting from survey to $3 \mathrm{D}$ modeling, the best result about geometrical fidelity, precision data, overall accuracy and visual experience.

For these reasons, multiple surveying techniques were employed in order to produce a $3 \mathrm{D}$ reality-based model of the case study, according to its characteristics and to the project aims.

\subsection{Data acquisition}

The survey was carried out using a terrestrial laser scanner (TLS) to obtain a geometrically accurate 3D model. Given the morphology, materials and colors of the chapel, the geometrical data has been integrated with informations collected during a 
photogrammetric campaign and processed in compliance with state of the art techniques.

A Continuous Wave Faro Focus 3D S120 laser scanner, with a field of view of $360^{\circ} \times 305^{\circ}$ and an integrated camera, was used to perform a total number of 3 scans, at a medium resolution and quality, with a spatial resolution of $6 \mathrm{~mm}$ at $10 \mathrm{~m}$. These parameters were considered a sufficient sampling step for the case study whose spatial configuration did not involve particular planning problems for the tangency of the surfaces. Starting from the entrance, the positions of the different acquisitions have been organised to cover the entire volume of the nave, taking into account any shadows produced by the presence of the statue of the veiled Christ. Whereas the focus of the study is on the coverage system, this latter aspect was not considered relevant. Moreover, due to the features of the chapel, no planar printed checkboards targets and spheres were necessary for the alignment procedure.

A terrestrial photogrammetric survey - focused on the roofing vault - was carried out mainly for texturing purposes but also to integrate the data missing in the laser scanning 3D survey. Using a Reflex Canon EOS 1300D and a zoom 18-55 lens set at $24 \mathrm{~mm}$ view, about 130 images were acquired planning a mean GSD of less than $4 \mathrm{~mm}$, considering the maximum distance obtainable from the object. A mean overlap of about $70 \%$ was chosen. No radiometric pre-processing of the acquired raw images was necessary considering the uniform lighting conditions during the survey.

\subsection{Data processing}

The complete range-based 3D point cloud was obtained employing a classical processing procedure. The adjacent TLS stations were aligned within the proprietary software Faro Scene, using a solid-rigid transformation based on cloud to cloud alignment procedure. A final point cloud of about 130 millions points was obtained.

At the same moment, the images were automatically oriented in the state-of-the-art Structure from Motion (SfM) software application Agisoft Metashape. The photogrammetric point cloud - about 8,700,000 points - was scaled using a known distance measured in the field. In order to produce a physically accurate model that also delivers a photorealistic view of the surveyed cultural site, the collected data were integrated. So the TLS and photogrammetric point clouds have been aligned and combined choosing the laser scanning coordinate system as reference and applying a similarity transformation.

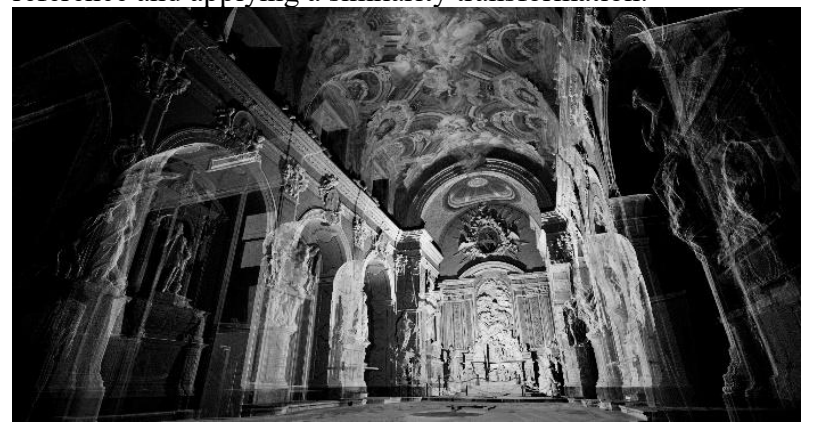

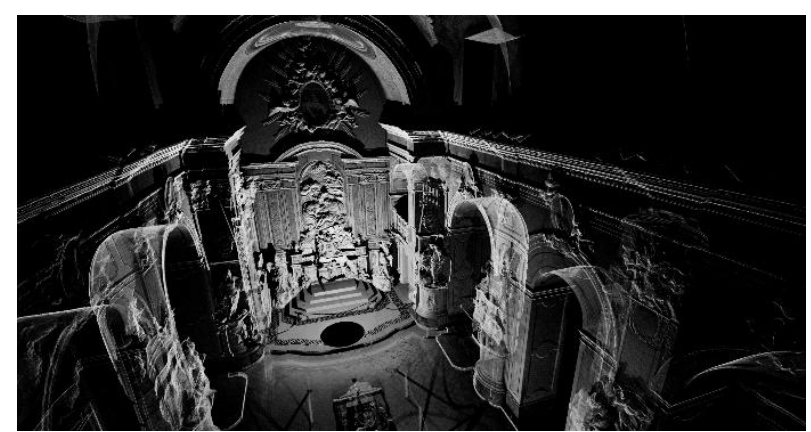

Figure 3. TLS point cloud
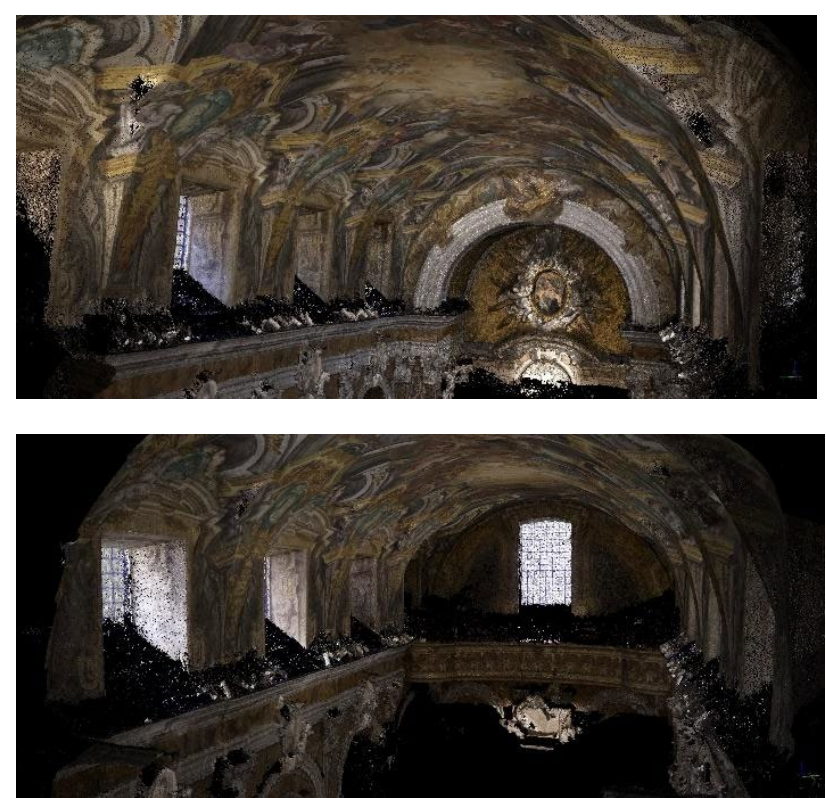

Figure 4. Photogrammetric point cloud

The final RMSE of the alignment resulted of $0.02 \mathrm{~m}$. The merged cloud was triangulated in order to obtain a reality-based 3D model.

A polygonal mesh model was generated using the Poisson surface reconstruction algorithm implemented in CloudCompare. Set an appropriate octree level value for the desired mesh resolution, a polygonal model of about 21 million of triangles was processed. Considering the research's aims, the model was simplified using a successive geometric optimisation. The quadratic edge collapse algorithm was applied and a final mesh of about 1 million of triangles was produced.

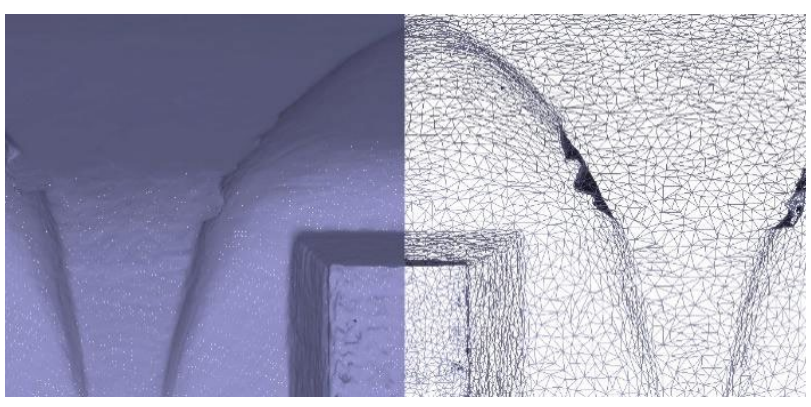

Figure 5. Comparison between the high-poly mesh (on the left) and the decimated one (on the right). Detail of a window 
The deviation between the original and the decimated mesh was measured by calculating the Hausdorff distance. The approximation error was below $1 \mathrm{~cm}$. Lastly, the decimated mesh model was textured using the images acquired for the photogrammetric survey thus obtaining better color information.

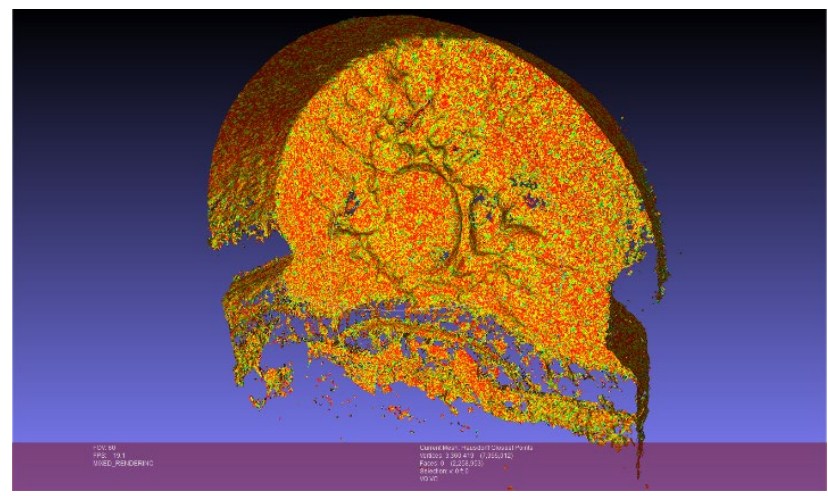

Figure 6. Check error simplification by calculating the Haursdorff distance. Detail of the apse

\section{PROJECTION / PERCEPTION}

In general, the visual perception we have of spaces is always linked to the conical perspective. Any other point of view would be related to a more technical aspect that has nothing to do with reality. The axonometric perspectives or orthogonal views of an object are tools that we have developed to understand the real shape of objects and know their exact dimensions.

In this case Francesco Maria Russo made the fresco Gloria del Paradiso or Paradiso dei di Sangro, which adorns the barrel vault that covers Sansevero's chapel, it was done thinking about the perspective point from the visitor's position. It is a painting in which you can only perceive the perspective it represents, from a single point of the church, and this corresponds to the central area of the nave. This painting can also be observed from other points inside the temple, such as the feet of the church or from the head, but the way of perceiving the geometry changes completely. Even from some points we get to see deformations in the geometry. Francesco, in order to develop a painting that was visualized with a correct perspective from the ground, had previously had an idea of the surface on which he was going to work, since his canvas was not a flat surface but a curved surface. This makes their development even more difficult, since they have to introduce small deformations so that the elements that they represent in perspective are visualized with a correct form. We have a space with a smooth surface in which Francesco applies the painting simulating shapes and reliefs that do not really exist, giving the space a depth greater than what it actually has. Observing the fresco well we can detect that in the central part the figures and geometries are flatter, while as the cornice approaches the extremes the perspective is more accentuated.

The aspect of the painting Glory of Paradise is known for the conical perspective in which it is drawn, but our goal was to achieve a different point of view. To do this we performed a process of extraction of the envelope from the three-dimensional model that we had obtained through the use of TLS and digital photogrammetry.

In architecture, when we want to represent a plan view or an elevation of an object, we resort to the dihedral projection making an orthogonal projection. This way of representing allows us to see an object in true magnitude in all its axes. This works well when they are flat surfaces, but the same is not true for curved surfaces.
When we perform an orthogonal projection of a curved surface, we obtain a drawing in which on one of the axes the measurement is proportional, but on the other axis it is not real but there are deformations. In this case, the barrel vault looks like a rectangle that matches the shape in plan. However, if we deploy the $3 \mathrm{~d}$ development of the cylinder to a flat element, that rectangle is not proportional to the plan projection, but is deformed, not in length but in width.

To unfold the vault in a flat projection, what we do is to open the envelope, taking as reference the virtual axis that coincides with the center of the cylinder. This type of graphic representation is known as cylindrical projection.

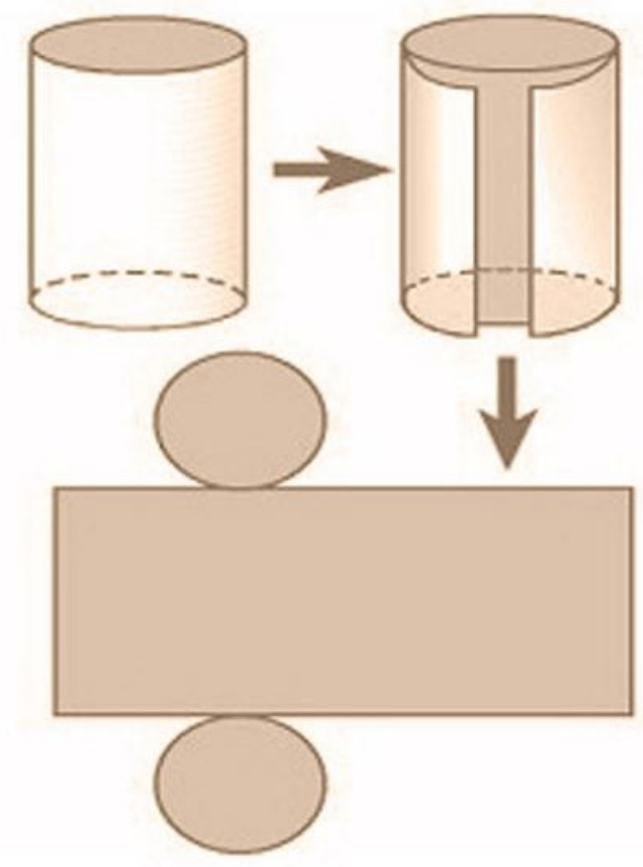

Figure 7. Process of unfolding the envelope of a cylinder in a flat projection

Carrying out the development of the geometry of a $3 \mathrm{~d}$ element is easy, but we are more interested in realizing it in the real texture to be able to visualize the deformations that exist in the painting and that we can only see doing this process. For this reason, the 3D model we have obtained, using three-dimensional scanning techniques by photogrammetry of the laser scanner, is a model of triangles with a geometry and a very precise photographic texture that fits perfectly with reality.

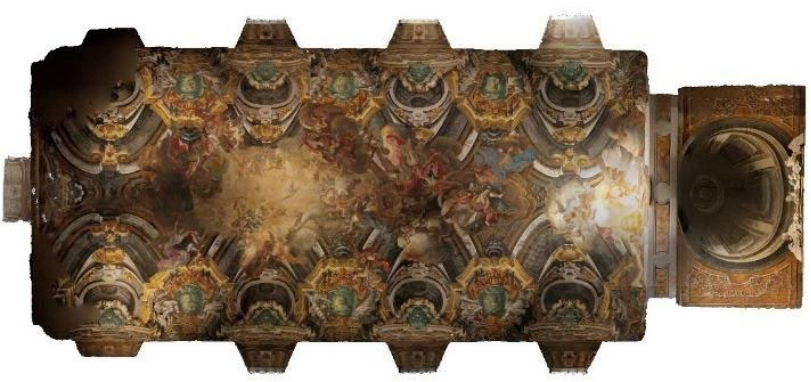

Figure 8. Orthogonal projection of the geometry of the vault and the paintings that decorate it 
To achieve this development, we use Agisoft Metashape with the objective of reprojecting the texture on the flat development of the curvature of the vault. The geometric result is a flat triangular mesh, but which coincides with the envelope of that vault and which contains the actual color values from the reprojection of the photographic texture. With this we can export the flat projection resulting from the deployment of the vault by means of a mathematical algorithm, which allows us to observe that the painting in the central part is not deformed, while in the areas near the cornice if they suffer deformations.

The most prominent visual differences between the flat projection of the vault and the flat projection of the vault's envelope can be seen in the extremes, but also in the geometries or circular forms, since in the first they are perfect circles, while in the second they are transformed into elliptical elements, which favor the visualization of perspective.

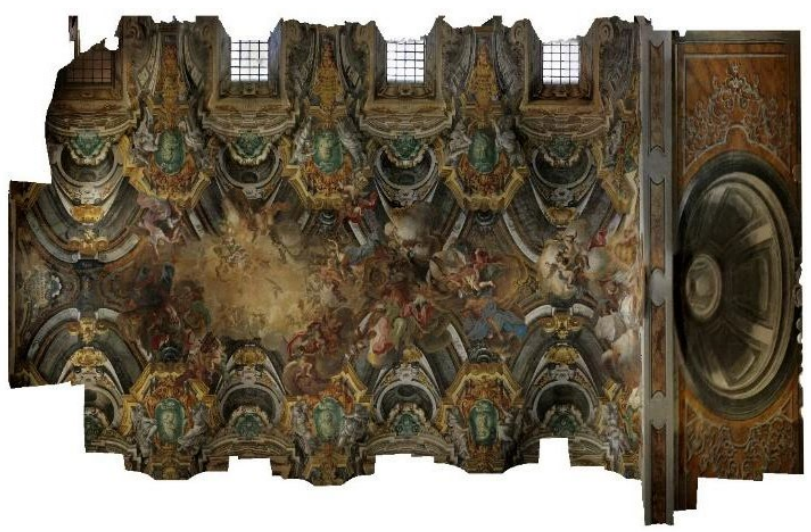

Figure 9. Cylindrical projection of the geometry of the vault and the paintings that decorate it
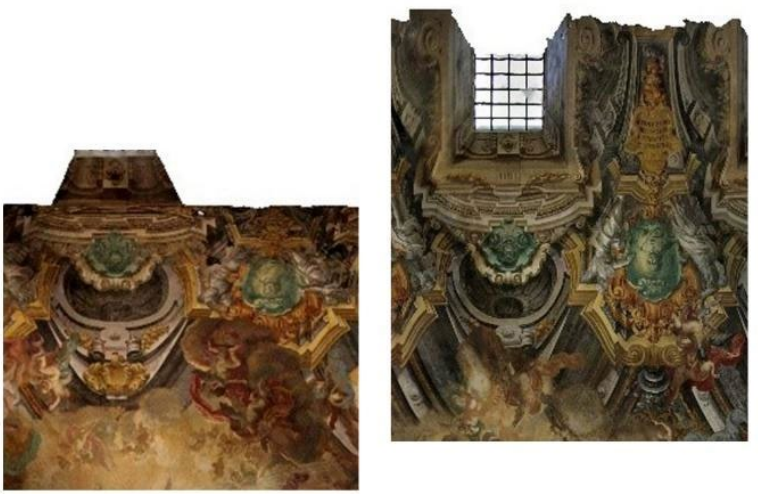

Figure 10. Difference between orthogonal projection and cylindrical projection

\section{CONCLUSIONS}

The use of three-dimensional digitalization tools, such as TLS or photogrammetry, allows to obtain very accurate and precise models of real geometries without being intrusive in the original objects. These techniques facilitate access to spaces, which due to their nature or conservation status, would otherwise be very difficult to study, as well as greatly speed up the time taken to collect data. These 3D models, having a great geometric and radiometric precision, allow us to carry out multiple studies on geometric deformations, the detection of pathologies, etc.
Obtaining a $3 \mathrm{~d}$ model of the vault that is so reliable to the original and thanks to the new tools of graphic computing, has allowed us to obtain a new point of view of the fresco of Gloria del Paradiso. On the one hand we have the point of view of the conical perspective, which as visitors we have when viewing the work. But on the other hand, we have the most technical point of view that the orthographic or flat projection offers us. This point of view makes it easier for us to measure and have exact geometry data, although in our case, being a flat projection of a curved surface, there are deformations at the ends where the measurements are not precise. For this reason, we have gone a little further, looking for a new orthogonal projection, geometrically improved, through the development in flat view of the envelope of the cylinder that defines the vault. This is a point of view in which any deformation is completely eliminated, and everything we visualize is in true magnitude.

\section{ACKNOWLEDGEMENTS}

The authors are thankful to the Museo Cappella Sansevero for permitting the data acquisition.

\section{REFERENCES}

Bracci, F., Drauschke M., Kühne S. and Márton Z.-C., 2018. Challenges in fusion of heterogeneous point clouds. ISPRS Int. Arch. Of Photogrammetry Remote Sensing and Spatial Informations Sciences, 42(2), pp. 155-162.

Cabeleira, J., 2019. Deconstructing the Imaginary Space of a Quadratura. Nexus Network Journal, pp. 1-20.

Calantropio, A., Chiabrando, F., Rinaudo, F. and Losè, L. T., 2018. Use and evaluation of a short range small quadcopter and a portable imaging laser for built heritage 3D documentation. ISPRS Int. Arch. of Photogrammetry, Remote Sensing and Spatial Information Sciences, 42(1), pp. 71-78.

Cera, V., Campi, M., 2017. Evaluating the potential of imaging rover for automatic point cloud generation. ISPRS Int. Arch. of Photogrammetry, Remote Sensing and Spatial Information Sciences, XLII-2/W3, pp. 147-154.

Di Paola, F., 2014. Metodologie di indagine applicate alla prospettiva solida nell'architettura in Sicilia. Un caso di studio: l'Oratorio di San Lorenzo a Palermo. In: Valenti, G. M. (eds), Prospettive architettoniche. Conservazione digitale, divulgazione e studio, vol. I, Sapienza Università Editrice, pp. 8194.

Farella, E. M., Torresani, A. and Remondino, F., 2019. Quality features for the integration of terrestrial and UAV images. ISPRS Int. Arch. of Photogrammetry, Remote Sensing and Spatial Information Sciences, XLII-2/W9, pp. 339-346.

Giordano, A., Nichele, C., 2016. L'Architectura Picta e la realizzazione della chiesa ideale a pianta centrale: il ruolo della rappresentazione per la conoscenza, l'elaborazione e la comunicazione tra ricerca e fruizione turistico-culturale. In: Bertocci, S., Bini., M. (eds), Le ragioni del disegno. Pensiero, Forma e Modello nella Gestione della Complessità, Gangemi editore, pp. 353-358.

Hoon Jo, Y., Hong, S., 2019. Three-Dimensional Digital Documentation of Cultural Heritage Site Based on the Convergence of Terrestrial Laser Scanning and Unmanned 
Aerial Vehicle Photogrammetry. International Journal of GeoInformation, 8(2), 53, $14 \mathrm{pp}$.

Liang, H., Li, W., Lai, S., Zhu, L., Jiang, W. And Zhang, Q., 2018. The integration of terrestrial laser scanning and terrestrial and unmanned aerial vehicle digital photogrammetry for the documentation of Chinese classical gardens - A case study of Huanxiu Shanzhuang, Suzhou, China. Journal of Cultural Heritage, 33, pp. 222-230.

Ogawa, T., Hori, Y., 2019. Comparison with accuracy of terrestrial laser scanner by using point cloud aligned with shape matching and best fitting methods. ISPRS Int. Arch. of Photogrammetry, Remote Sensing and Spatial Information Sciences, XLII-2/W9, pp. 535-541.

Pérez-Sinticala C., Janvier R., Brunetaud X., Treuillet S., Aguilar R. and Castañeda B., 2019. Evaluation of Primitive Extraction Methods from Point Clouds of Cultural Heritage Buildings. In: Aguilar R., Torrealva D., Moreira S., Pando M.A. and Ramos L.F. (eds) Structural Analysis of Historical Constructions. RILEM Bookseries, vol 18. Springer, Cham.

Remondino F., Nocerino E., Toschi I. And Menna F., 2017. A critical review of automated photogrammetric processing of large datasets. ISPRS Int. Arch. of Photogrammetry, Remote Sensing and Spatial Information Sciences, 42(2/W5), pp. 591-599.

Rossi, M., Mele, G., Buratti, G., 2018. La prospettiva come architettura immateriale. Il finto coro di Santa Maria presso San Satiro a Milano. In: Salerno, R. (ed), Rappresentazione materiale/immateriale, Gangemi editore, pp. 215-224.

Serna, C. G., Pillay, R., Tremeau, A., 2015. Data fusion of objects using techniques such as laser scanning, structured light and photogrammetry for Cultural Heritage applications. Computational Color Imaging, pp. 208-224.

Toschi, I., Remondino, F., Rothe, R., and Klimek, K., 2018. Combining airbone oblique camera and lidar sensors: investigation and new perspectives. Int. Annals of Photogrammetry, Remote Sensing and Spatial Information Sciences, XLII-1, pp. 437-444.

Zieher, T., Toschi, I., Remondino, F., Rutzinger, M., Kofler, Ch., Mejia-Aguilar, A. and Schlögel, R., 2018. Sensor- and sceneguided integration of TLS and photogrammetric point clouds for landslide monitoring. ISPRS Int. Arch. of Photogrammetry, Remote Sensing and Spatial Information Sciences, 42(2), pp. 1243-1250. 